\title{
The Application of Methylprednisolone Nanoscale Zirconium-Porphyrin Metal-Organic Framework (MPS-NPMOF) in the Treatment of Photoreceptor Degeneration
}

This article was published in the following Dove Press journal:

International Journal of Nanomedicine

Yajie Wang, I,2,* Wei Liu, (iD) $3,4, *$ Bo Yuan, ${ }^{1,2}$ Xuebo Yin, ${ }^{3}$

Yiming Li, ' Zongjin Li, (D) Jianlin Cui,' Xiaoyong Yuan, ${ }^{2}$

Yuhao Li (iD) 1,5

'Tianjin Key Laboratory of Tumor Microenvironment and Neurovascular Regulation, Nankai University School of Medicine, Tianjin 30007I, People's Republic of China; ${ }^{2}$ Tianjin Eye Hospital, Tianjin Key Laboratory of Ophthalmology and Visual Science, Nankai University Eye Hospital, Tianjin 300020, People's Republic of China; ${ }^{3}$ State Key Laboratory of Medicinal Chemical Biology, Tianjin Key Laboratory of Biosensing and Molecular Recognition, Nankai University College of Chemistry, Tianjin 30007I, People's Republic of China;

${ }^{4}$ Tianjin University School of Science, Tianjin 300072, People's Republic of China;

${ }^{5}$ Tianjin Key Laboratory of Radiation Medicine and Molecular Nuclear Medicine, Tianjin 300 I92, People's Republic of China

*These authors contributed equally to this work

Correspondence: Yuhao Li

Tianjin Key Laboratory of Tumor

Microenvironment and Neurovascular

Regulation, Nankai University School of

Medicine, 94 Weijin Road, Tianjin 30007I,

People's Republic of China

Tel +8622 23502554

Email liyuhao@nankai.edu.cn

Xiaoyong Yuan

Tianjin Eye Hospital, Tianjin Key

Laboratory of Ophthalmology and Visual

Science, 4 Gansu Road, Tianjin 300020,

People's Republic of China

Tel +8622 2731 3336

Email yuanxy_cn@hotmail.com
Background: Photoreceptor degeneration is one of the most refractory oculopathy in the world, leading to vision loss in severe cases. Methyprednisolone is one of the most commonly prescribed medications for the treatment of retinal degenerative diseases, either by oral administration or repeated intraocular injections. However, the efficacy was unsatisfactory due to its systemic or local side effects and short retention time within the retina.

Methods: Nanoscale zirconium-porphyrin metal-organic framework (NPMOF) was synthesized and characterized. The biotoxicity and imaging capability of NPMOF were evaluated using zebrafish embryos and larvae. NPMOF was then used as a skeleton and loaded with methylprednisolone (MPS) to prepare a novel kind of nanoparticle, MPS-NPMOF. Photoreceptor degeneration was induced by high-intensity light exposure in adult zebrafish. MPS-NPMOF was delivered to the injured retina by intraocular injection. The photoreceptor regeneration and its underlying mechanism were explored by immunohistochemistry, quantitative real-time polymerase chain reaction and behavioral test.

Results: NPMOF not only had low biotoxicity but also emitted bright fluorescence. Following a single MPS-NPMOF intraocular injection, the injured retina exhibited the faster photoreceptor regeneration with better visual function by promoting the cell proliferation.

Conclusion: NPMOF is an ideal carrier and could be applied in tracking and delivering medications. By intraocular injection, the novel drug delivery system, MPS-NPMOF, accomplishes the sustained release of drug and plays a therapeutic role in photoreceptor degeneration. Keywords: nanoscale zirconium-porphyrin metal-organic framework, photoreceptor, degeneration, methylprednisolone, drug delivery

\section{Introduction}

Retinal degeneration is one of the leading causes of progressive blindness. In many human ocular diseases, such as retinitis pigmentosa and age-related macular degeneration, the death of photoreceptor cells was the principal event lacking curative treatment. ${ }^{1}$ Zebrafish is an excellent model of visual diseases, especially those involving dysfunction of photoreceptors, because their gene expression patterns, structure and function are remarkably similar to those of the human retina. ${ }^{2}$ The zebrafish model not only broadens our understanding of the genetics and signaling pathways underlying human ocular diseases but also provides novel insights for developing diagnostic and therapeutic approaches. 
Glucocorticoids are among the most commonly prescribed drugs for the treatment of retinal diseases because of their anti-inflammatory, immunosuppressive and vasoconstrictive effects by systemic administration or intravitreous injection. ${ }^{3-5}$ Unfortunately, the use of glucocorticoids is limited by their undesirable effects. ${ }^{6,7}$ Therefore, there is an urgent need to optimize medication delivery. In the intraocular system, some nanoparticles can protect their cargo from rapid degradation, sustain the release period and penetrate physiological barriers, thus delivering the loaded drugs to specific tissues and cells by either passive or active targeting mechanisms. ${ }^{8}{ }^{8}$ Porphyrin metal-organic frameworks (MOFs) are a class of porous materials composed of metal ions and organic ligands. On the one hand, these frameworks possess a large surface area, tunable porosity and robust stability. Their ultra-large channels can carry polycyclic drugs. ${ }^{10}$ On the other hand, they retain the photophysical properties of porphyrin and emit bright red fluorescence, which offers an approach to monitor and track the particles. ${ }^{11}$ Until now, little was known about the application of MOFs as intraocular carriers.

In this study, a nanoscale zirconium-porphyrin metalorganic framework (NPMOF) was synthesized and used as a skeleton. Methyprednisolone (MPS), a glucocorticoid medication, was then loaded to prepare a novel nanoparticle, MPS-NPMOF. Specifically, the following properties were determined: 1) physical and chemical characteristics of NPMOF; 2) in vivo biotoxicity and biodistribution of NPMOF; 3) uptake-release properties of MPS-NPMOF; and 4) the degeneration-regeneration of photoreceptors and functional changes following intraocular injection of MPS-NPMOF. To our knowledge, the present study is the first report on an NPMOF-based intraocular delivery system and creates an avenue to develop a new therapeutic approach for photoreceptor degeneration.

\section{Materials and Methods}

\section{Synthesis and Characterization of NPMOF}

NPMOF was synthesized by a slightly modified solventthermal method. ${ }^{11}$ Briefly, $10 \mathrm{mg}$ of 5,10,15,20-tetrakis(4-carboxyl)-21H,23H-porphine (TCPP; TCI Shanghai Chemical Industry, China), $30 \mathrm{mg}$ of zirconium chloride $\left(\mathrm{ZrCl}_{4}\right.$; AlfaAsia, Tianjin, China), $200 \mathrm{mg}$ of benzoic acid (BA, 98.5\%; Guangfu Fine Chemical Research Institute, Tianjin, China), $100 \mathrm{mg}$ of cetyltrimethylammonium bromide (CTAB; Fuchen Reagent, Tianjin, China), $200 \mathrm{mg}$ of polyethylene glycol M.W.
6000 (PEG-6000; Fuchen Reagent), and $10 \mathrm{~mL}$ of dimethylformamide (DMF; Concord Reagent, Tianjin, China) were mixed and dissolved by ultrasonication for 10 mins. The dark purple suspension was heated at $120^{\circ} \mathrm{C}$ for $24 \mathrm{hrs}$ in a 30 $\mathrm{mL}$ Teflon-lined steel-autoclave and subsequently cooled to room temperature. NPMOF was purified by centrifugation, washed with DMF, $100 \%$ ethanol, and $\mathrm{ddH}_{2} \mathrm{O}$ twice each and then dried by lyophilization. To obtain a $1 \mathrm{mg} / \mathrm{mL}$ NPMOF solution, NPMOF was re-suspended in $0.1 \mathrm{M}$ phosphate buffered saline (PBS, pH 7.4; Sangon Biotech, China).

The morphology of NPMOF was observed using transmission electron microscopy (TEM, accelerating voltage $=200 \mathrm{kV}$; Tecnai $\mathrm{G}^{2}$ F20, FEI, Hillsboro, USA) and scanning electron microscopy (SEM; JSM-7500F, JEOL, Tokyo, Japan). The structure of NPMOF was characterized by X-ray diffraction (XRD), $\mathrm{N}_{2}$ adsorption-desorption and pore-size distribution. XRD patterns were obtained by a $\mathrm{D} /$ max-2500 diffractometer (Rigaku, Tokyo, Japan) using $\mathrm{Cu}-\mathrm{K} \alpha$ radiation $(\lambda=1.5418 \AA) . \mathrm{N}_{2}$ adsorption-desorption isotherms and the pore size distribution were tested with a Tristar 3000 at $77 \mathrm{~K}$. The pore-size distribution was calculated by the Barrett, Joyner and Halenda (BJH) method according to the density functional theory (DFT) program. The composition of NPMOF was examined by thermogravimetric analysis (TGA), differential scanning calorimetry (DSC) and Fourier transform infrared (FTIR) spectroscopy (400-4000 $\mathrm{cm}^{-1}$ ). TGA was performed on a PTC-10ATG-DTA analyzer (Thermo plus EVO2 TG8121, Rigaku) heated from $20^{\circ} \mathrm{C}$ at a ramp rate of $10^{\circ} \mathrm{C} / \mathrm{min}$ under air, and infrared spectra were obtained via a Bruker TENSOR 27 Fourier transform infrared spectrometer. The optical properties of NPMOF were revealed using a UV-2450-visible spectrophotometer (Shimadzu, Japan) and FL-4600 Fluorescence Spectrometer (Hitachi, Japan) equipped with a quartz cell $(1 \mathrm{~cm} \times 1 \mathrm{~cm})$.

\section{Preparation of MPS-NPMOF}

To prepare loaded nanoparticles, $10 \mathrm{mg}$ of methylprednisolone (MPS; Adamas, China) was mixed with $5 \mathrm{mg}$ of NPMOF in a $10 \mathrm{~mL}$ DMF solution under stirring for three days. After 2, 4, 8, 12, 24, 48, and $72 \mathrm{hrs}$ of agitation, $0.5 \mathrm{~mL}$ of solution was centrifuged, and the supernatant was diluted and analyzed by UV-Vis spectrophotometry at $266 \mathrm{~nm}$. The loading efficiency $(L E)$ was calculated with the following equation:

$$
L E=\frac{W_{D}}{W_{M O F}} \times 100 \%
$$


where $D$ indicates the drug. $W_{D}$ is the mass of MPS loaded in the NPMOF, and $W_{M O F}$ is the original mass of NPMOF. $W_{D}$ was calculated from the adsorption value at $266 \mathrm{~nm}$.

After $72 \mathrm{~h}$, the remaining mixture was centrifuged, dried and re-suspended in $0.1 \mathrm{M}$ PBS to a concentration of $2 \mathrm{mg} / \mathrm{mL}$ MPS-NPMOF.

MPS release experiments were conducted by incubating $5 \mathrm{mg}$ of MPS-NPMOF in $5 \mathrm{~mL}$ of $5 \%$ hyaluronic acid solution for three days to simulate the environment of the vitreous body. The amounts of released MPS in the supernatant were measured by fluorescence spectrophotometry at $430 \mathrm{~nm}$ after $4,8,12,24,48$, and $72 \mathrm{hrs}$ of agitation. The release efficiency $(R E)$ was calculated with the following equation:

$$
R E=\frac{W_{R E}}{W_{D}} \times 100 \%
$$

where $W_{R E}$ represents the mass of MPS released from MPS-NPMOF. $W_{D}$ means the mass of MPS loaded into NPMOF.

\section{Experimental Animals}

Wild-type zebrafish (AB strain) and transgenic zebrafish $\mathrm{Tg}$ (lysC:EGFP) (a gift from Dr. Yao) were used in this study. The animals were maintained in a fish facility under standard conditions, with a $10 / 14 \mathrm{hr}$ dark/light cycle at $28.5^{\circ} \mathrm{C} .{ }^{12}$ Protocols for all animal procedures were approved by the Nankai University Animal Care and Use Committee and complied with National Institutes of Health (NIH) guidelines.

\section{Aqueous Exposure}

At 48 hrs post fertilization (hpf), wild-type and $\operatorname{Tg}$ (lys $C$ : EGFP) embryos were placed in a 6-well plate (30 embryos per well) and exposed continuously to NPMOF-Holt buffer $\left(60 \mathrm{mmol} / \mathrm{L} \mathrm{NaCl}, 0.67 \mathrm{mmol} / \mathrm{L} \mathrm{KCl}, 0.3 \mathrm{mmol} / \mathrm{L} \mathrm{NaHCO}_{3}\right.$, $0.9 \mathrm{mmol} / \mathrm{L} \mathrm{CaCl}, \mathrm{pH} 7.2$ ) at a concentration of $100 \mathrm{mg} / \mathrm{L}$ and $50 \mathrm{mg} / \mathrm{L}$, respectively, until 120 hpf. Thirty embryos were raised in Holt buffer as the control group. The experiment described above was repeated three times.

\section{Light Lesion and Intravitreous Injection}

To induce selective photoreceptor death, adult wild-type fish were exposed to high-intensity light from a mercury arc lamp (>180,000 lux, BH2-RFL-T3-W, Olympus, Japan) for 45 mins. ${ }^{13}$ Thirty minutes after light lesioning, the fish were anesthetized with $0.1 \%$ 3-aminobenzoic acid ethyl ester methanesulfonate (MS-222; Sigma, St. Louis, MO, USA). An incision was made in the corneal limbus using a microblade. In all experiments except behavioral test, only the left eye was injected while the right eye was used as the uninjected control. A microsyringe (Hamilton, 1701RN; Reno, Nevada, USA) was used to operate injection, $0.8 \mu \mathrm{L}$ of either control (0.1 M PBS, pH 7.4) or 0.1 M PBS containing $1 \mathrm{mg} / \mathrm{mL}$ MPS, $1 \mathrm{mg} / \mathrm{mL}$ NPMOF, or $2 \mathrm{mg} / \mathrm{mL}$ MPSNPMOF into the vitreous chamber. Following injection, the fish were revived in fish water, returned to the system and raised regularly. Thirty fish were injected in each group.

\section{Histology and Immunofluorescence}

NPMOF-exposed larvae at $120 \mathrm{hpf}$ or injected adult fish were anesthetized in $0.1 \%$ MS-222 and euthanized immediately. In adult fish, eye cups were harvested at 1, 2, 3, 4, 5, 6 and 7 days post lesion (dpl). Larvae or eye cups were fixed in 4\% paraformaldehyde (PFA). Hematoxylin and eosin (HE) staining and immunohistochemistry were performed using standard procedures. ${ }^{14,15}$ The primary antibodies were Zpr1 (1:200; Zebrafish International Resource Center (ZIRC), Eugene, OR, USA), Zpr3 (1:200; ZIRC), Zn12 (1:200; ZIRC) and anti-proliferating cell nuclear antigen (PCNA, 1:1000; clone PC-10, Sigma). For PCNA immunolabelling, slides were first incubated in $0.01 \mathrm{M}$ sodium citrate buffer ( $\mathrm{pH}$ 6.0) with $0.05 \%$ Tween-20 at $95-98^{\circ} \mathrm{C}$ for $20 \mathrm{~min}$ prior to retrieving the antigen. ${ }^{16}$ The secondary antibody was Alexa Fluor 488 (1:1000; ab150113, Abcam, Cambridge, MA) or fluorescent-labelled Сy3 (1:500; Millipore, Billerica, MA). The sections were counterstained with a 1:1000 dilution of 4',6-diamidino-2-phenylindole (DAPI, Sigma) to label the nuclei.

\section{In situ Hybridization}

Embryos were raised in 0.003\% 1-phenyl-2-thiourea (PTU, Sigma) until $24 \mathrm{hpf}$ to prevent pigmentation until $120 \mathrm{hpf}$. Larvae were anesthetized with $0.1 \%$ MS-222, euthanized immediately and fixed in 4\% PFA at $120 \mathrm{hpf}$. Hepatocytes were specifically labeled by ceruloplasmin ( $c p$, GenBank NM_131802) mRNA probe. The primer sequences for the amplification of $c p$ were forward 5'-TGGAACGCTCTA CCACAA- $3^{\prime}$ and reverse 5'-TACGGTTCTGTGGAATAG TG-3'. The cDNA was linearized with Sac I, and the digoxigenin-labeled RNA probe was synthesized with T7 polymerase. Whole-mount in situ hybridization was performed using a standard protocol. ${ }^{17}$ The concentration of $c p$ probe was $2 \mathrm{ng} / \mu \mathrm{L}$. Six individuals were processed in each group. 


\section{Quantitative Real-Time Polymerase Chain Reaction (qRT-PCR)}

Total RNA was extracted from larval zebrafish at $120 \mathrm{hpf}$ or eye cups using TRIzol reagent according to the manufacturer's protocol (Life Technologies, Carlsbad, California, USA) and was reverse-transcribed using TransScript FirstStrand cDNA Synthesis SuperMix (TransGen Biotech, Beijing, China). qRT-PCR was assembled using TransStart Top Green qPCR SuperMix (TransGen). Reaction procedures were as follows: 5 mins at $95^{\circ} \mathrm{C}$, followed by 40 cycles of 15 seconds at $95^{\circ} \mathrm{C}, 20$ seconds at $55^{\circ} \mathrm{C}$, and 30 seconds at $72^{\circ} \mathrm{C}$. Primer sequences are listed in Supplementary Table 1. The comparative $2^{-\Delta \Delta \mathrm{Ct}}$ method was used for data analysis. The qRT-PCR experiment described above was repeated 3 times.

\section{Behavioral Test}

Optomotor response (OMR) tests were performed on adult zebrafish from the normal (unlesioned and uninjected), $0.1 \mathrm{M}$ PBS, $1 \mathrm{mg} / \mathrm{mL}$ MPS and $2 \mathrm{mg} / \mathrm{mL}$ MPS-NPMOF groups at $7 \mathrm{dpl}$. The injection was operated biocularly as described previously. The injectants were same in both eyes. The normal group was used to determine the baseline of parameters. All behavioral tests were performed between 14:00 and 18:00 to avoid the influence of circadian rhythms on locomotor activity and visual sensitivity. $^{18,19}$ The stimulus of the OMR apparatus was a rotating, radial, black and white grating, modified from a classic OMR stimulus. ${ }^{20,21}$ The spatial and temporal frequencies were set at 0.03 cycles per degree (c/deg) and 30 rotations per minute (rpm), respectively. After a 15 min acclimatization, the movement was tracked using a camera (CX240, Sony, Japan) positioned above the apparatus. The grating was presented to the fish in four 60 -second trials, with the first two trials serving as adaptation and the last two trials used for data collection. For each pair of trials, the gratings rotated once clockwise and once counterclockwise. All digital tracks were analyzed by Ethovision XT software (version 11.5, Noldus Information Technology, Wageningen, the Netherlands). The positive response was defined as swimming following the grating direction. OMR performance was quantified by the positive proportion of distance (distance of positive response/ total distance) and positive proportion of time (time of positive response/total time). Eight fish were tested in each group.

\section{Photography and Image Analysis}

Images of immunofluorescence were captured with an LSM710 confocal microscope (Carl Zeiss, Jena, Germany). Images of HE staining, in situ hybridization, NPMOF distribution and neutrophil aggregation were photographed with a BX51 microscope (Olympus Corporation, Tokyo, Japan). ImageJ software (1.49X, NIH, http://rsb.info.nih.gov/ij/) was used to convert images to 8-bit grayscale prior to thresholding; subsequently count GFP-expressing and retinal cells; calculate the positive areas of Zpr1, Zpr3 and Zn12 immunostaining; and measure the defect length of retinas following light lesioning.

\section{Statistical Analysis}

Statistical analysis was performed with SPSS software (version 20.0, IBM, New York, USA) and GraphPad software (version 5.0, GraphPad Software, La Jolla, USA). Statistical analysis of differences among groups was determined by using one-way analysis of variance (ANOVA) with the exception of the survival rate (with the Log rank test). The significance level was set at $p$-value $<0.05$.

\section{Results and Discussion \\ Characterization of NPMOF}

In the present study, we prepared a novel NPMOF and used it as a loading skeleton. The design strategy for NPMOF was as follows: BA and CTAB were used as subsidiary ligand blockers and surfactants to regulate the size. Zirconium was selected as the metal ion of NPMOF because of its high biocompatibility. The dispersibility and hydrophilicity of NPMOF were improved using PEG-6000. Porphyrin was used as the imaging unit because it can provide powerful red fluorescence. Figure 1A shows the schematic illustration of NPMOF. There were two main channels that could carry the drug. $\mathrm{Zr}_{6} \mathrm{O}_{16}$ clusters (green polyhedron) were linked through the planar TCPP to form a Kagome-type topology. TEM and SEM images revealed that NPMOF had a spherical structure approximately $270 \mathrm{~nm}$ in diameter (Figure 1B and $\mathrm{C}$ ), so the water-soluble NPMOF could easily enter into the zebrafish. ${ }^{11}$ The XRD study showed that the main diffraction peaks of NPMOF emerged at $2 \theta$ values of $4.7^{\circ}, 5.4^{\circ}$, $7.1^{\circ}, 9.4^{\circ}, 10.8^{\circ}, 11.8^{\circ}$ and $14.3^{\circ}$ (Figure 1D). These sharp and narrow peaks indicated that NPMOF was highly crystalline and finely porous. $\mathrm{N}_{2}$ adsorption-desorption isotherms of NPMOF were classified as type II according to International Union of Pure and Applied Chemistry (IUPAC) nomenclature (Figure 1E; see $^{22}$ ), indicating the presence of mesopores. 
A


H
B
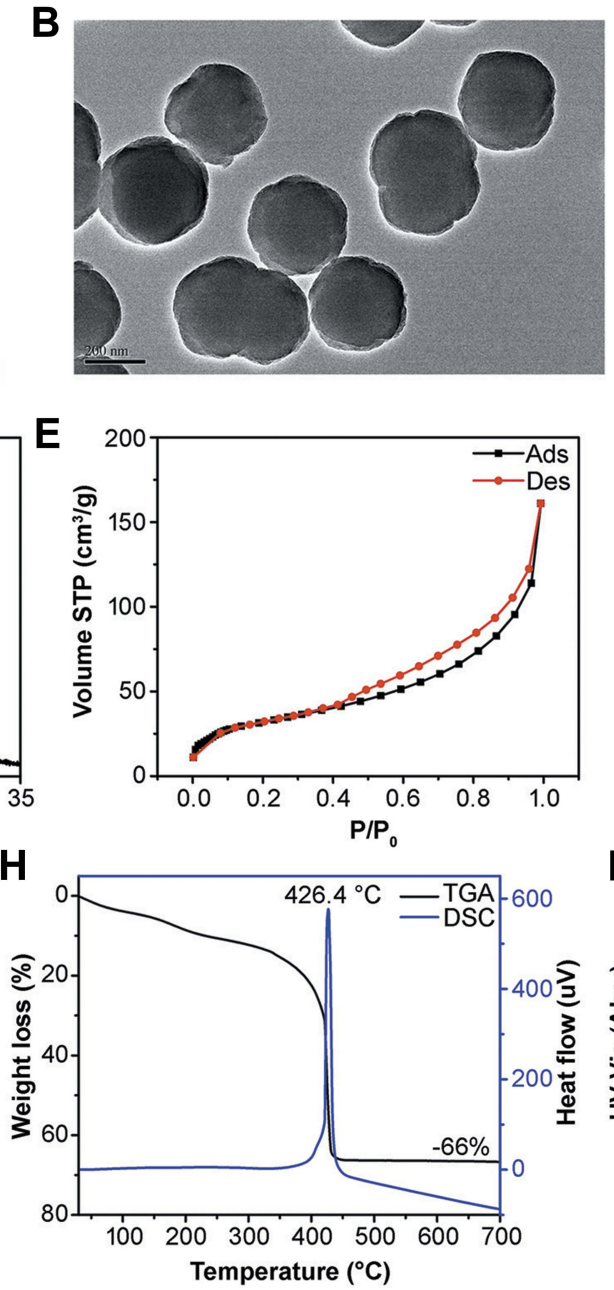

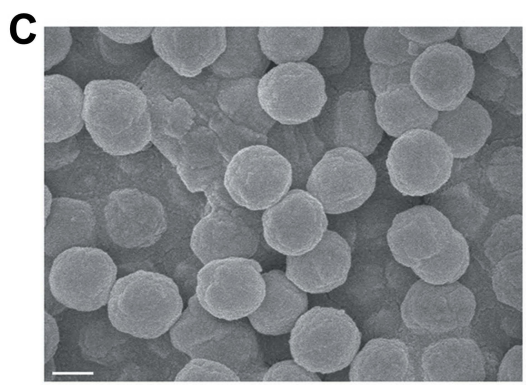

$F$

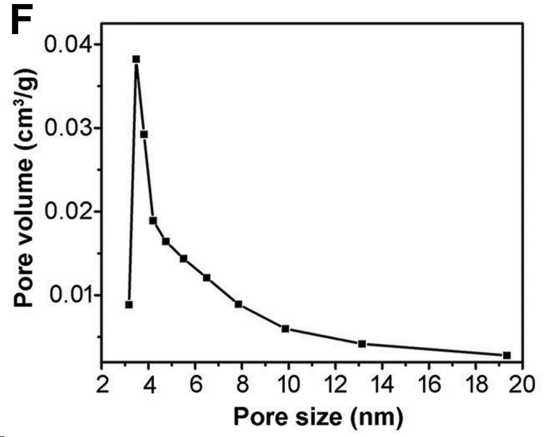

1

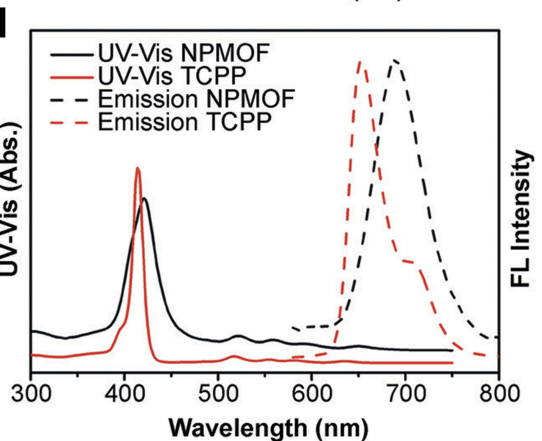

Figure I The morphology, composition and optical properties of NPMOF. (A) Schematic illustration of NPMOF. (B) TEM and (C) SEM images of NPMOF show an average size of $270 \mathrm{~nm}$. (D) XRD pattern, (E) adsorption and desorption isotherm, (F) pore size distribution, (G) FTIR curve and (H) TGA and DSC analysis of NPMOF. (I) UV-Vis and fluorescence spectra of TCPP (red, in pH $10 \mathrm{NaOH}$ aqueous solution, $25^{\circ} \mathrm{C} ; \lambda_{\mathrm{ex}}=514 \mathrm{~nm}$ ) and NPMOF (black, in water, $25^{\circ} \mathrm{C} ; \lambda_{\mathrm{ex}}=530 \mathrm{~nm}$ ). Note that compared to TCPP, the UV-Vis absorbance and fluorescence of NPMOF revealed a slight red-shift. Scale bar in (B) and (C): $200 \mathrm{~nm}$.

The Brunauer-Emmett-Teller (BET) surface area of NPMOF was $111.4 \mathrm{~m}^{2} \mathrm{~g}^{-1}$, and the main pore size was $3.5 \mathrm{~nm}$ (Figure 1F). The FTIR image showed a peak at $656 \mathrm{~cm}^{-1}$ that was assigned to the $\mathrm{Zr}-\mathrm{O}$ vibration of the $\mathrm{Zr}$ cluster (Figure 1G, arrow). The composition and stability of NPMOF were detected by TGA. DSC curves showed that NPMOF was stable until $426.4^{\circ} \mathrm{C}$, and the calculated molecular formula was $\mathrm{Zr}_{3} \mathrm{O}_{8}(\mathrm{OH})_{3}$ TCPP (Figure 1H). For UVVis adsorption, NPMOF exhibited a strong absorption peak at $421 \mathrm{~nm}$ for the Soret band and four peaks at 522, 560, 590, and $650 \mathrm{~nm}$ for the $\mathrm{Q}$ band, while fluorescence was observed at an emission peak at $689 \mathrm{~nm}$ (Figure 1I). Compared to the spectrum of the precursor porphyrin (TCPP), most peaks were still present, although slightly red-shifted (Figure 1I), which was attributed to the enlarged conjugate area of porphyrin after NPMOF formation. ${ }^{23}$

\section{Biological Distribution and Phenotypes Following NPMOF Exposure}

To observe the in vivo distribution of NPMOF, embryos were exposed to NPMOF until $120 \mathrm{hpf}$. Phenotypes were assessed on exposed larvae at 96 and $120 \mathrm{hpf}$. Compared to the control group, no obvious malformation was observed in embryos in the exposed groups at any time point (Figure 2A-D). From $96 \mathrm{hpf}$, bright red fluorescence was detected, mainly in the gastrointestinal tract, in both the $50 \mathrm{mg} / \mathrm{L}$ and $100 \mathrm{mg} / \mathrm{L}$ exposed groups, and it intensified with longer exposure time (Figure 2B and D, arrowheads). The NPMOF distribution in the gastrointestinal tract was attributed to swallowing and digestion. ${ }^{24}$ To explore whether NPMOFs can enter into the retina, we performed cryosections of embryos at $120 \mathrm{hpf}$. Compared to the control retina, scattered fluorescent signals were detected in the inner plexiform layer (IPL) and optic 


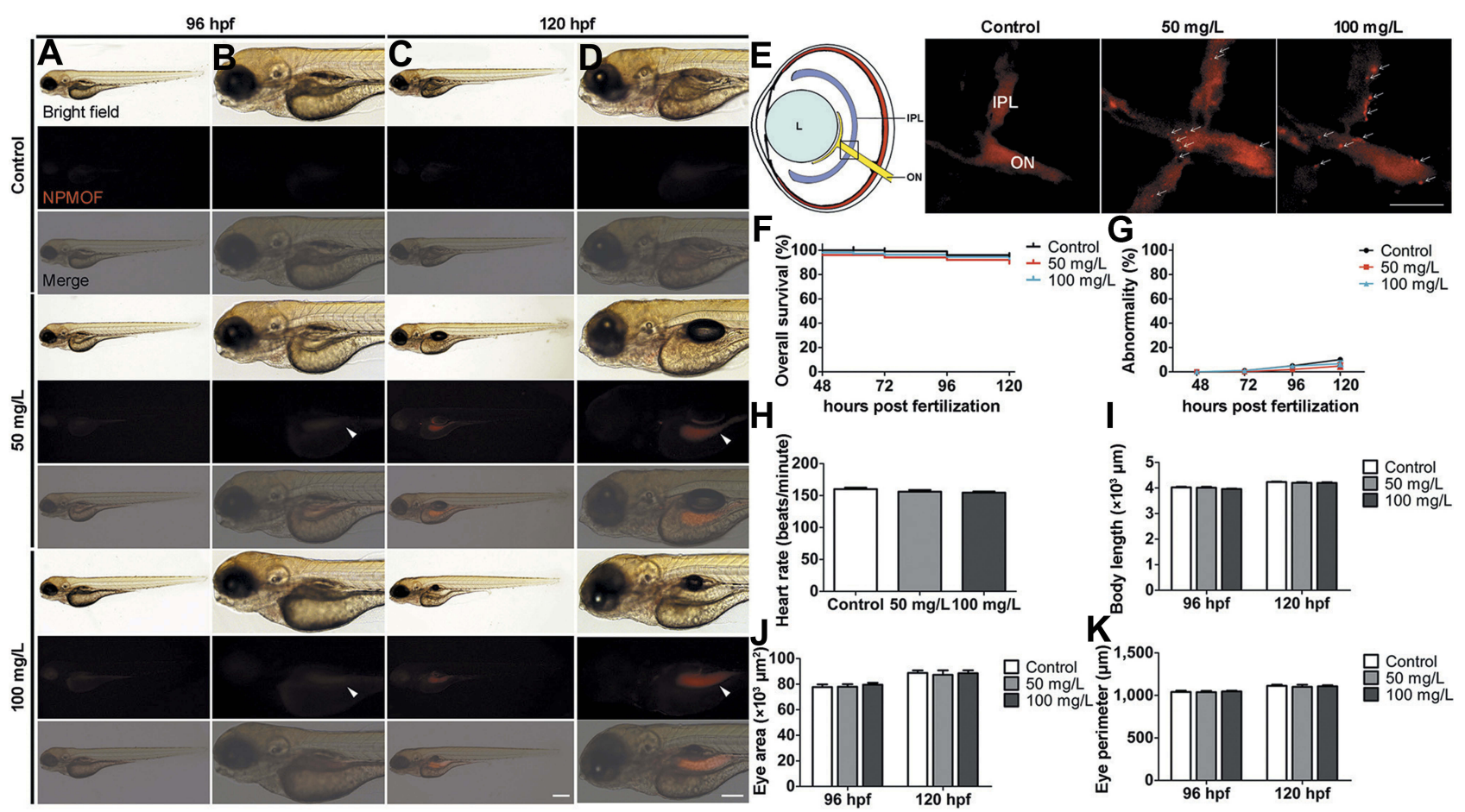

Figure 2 Phenotypes and tissue distribution of larval zebrafish following NPMOF exposure. Bright field and fluorescence images of larval zebrafish at (A and B) 96 and (C and D) 120 hpf. Columns (B) and (D) are magnified images of columns (A) and (C), respectively. Note that NPMOF gathers mainly in the gastrointestinal tract (arrowheads). (E) The NPMOF localization in sections taken from retinas in the control, $50 \mathrm{mg} / \mathrm{L}$ and $100 \mathrm{mg} / \mathrm{L}$ exposed groups at $\mathrm{I} 20 \mathrm{hpf}$. Note that NPMOF localizes predominantly in the IPL and ON (arrows). The location of the image is shown by the square in the cartoon. (F) Statistical analysis of the survival rate. No significant difference was found among the control, $50 \mathrm{mg} / \mathrm{L}$ and $100 \mathrm{mg} / \mathrm{L}$ exposed groups (Log rank test, $p>0.05$ ). (G) Statistical analysis of the malformation rate, $(\mathbf{H})$ heart rate at $120 \mathrm{hpf}$, (I) body length, (J) area and (K) perimeter of the eye ball at $96 \mathrm{hpf}$ and I20 hpf. No significant difference was found among the three groups (ANOVA, $p>0.05$ ). (A-D) Dorsal is up, and rostral is left. Scale bar in (A) and (C): $200 \mu \mathrm{m} ;(\mathbf{B})$ and (D): I00 $\mu \mathrm{m}$; (E): I0 $\mu \mathrm{m}$. Abbreviations: L, lens; IPL, inner plexiform layer; ON, optic nerve.

nerve $(\mathrm{ON})$ in the exposed groups (Figure 2E). The residence in eyeballs can be explained by nanoscale size and nutrient requirement of NPMOFs. Nanoparticles can penetrate across conjunctiva or skin and reach the retina. ${ }^{25}$ Moreover, porphyrin is one of the key components for many enzymes and proteins such as hemoglobin and $\mathrm{P} 450 .{ }^{26}$ Porphyrin is essential for biosynthesizing hemoglobin and is thus involved in erythropoiesis, oxygen carrying and the homeostatic balance of systems, including the nervous system. ${ }^{27-29}$ We then analyzed the survival rate, malformation rate, heart rate, body length and area and perimeter of the eye to further evaluate the biotoxicity (Figure $2 \mathrm{~F}-\mathrm{K}$ ). There was no significant difference among the control, $50 \mathrm{mg} / \mathrm{L}$ and $100 \mathrm{mg} / \mathrm{L}$ exposed groups (Figure $2 \mathrm{~F}-\mathrm{K}$; Log rank test \& ANOVA, $p>0.05$ ). The above data suggest that exposure to NPMOF does not lead to abnormal development.

\section{Retinal Neural Differentiation and Liver Development Following NPMOF Exposure}

To investigate whether NPMOF is neurotoxic, retinal histology and neural differentiation were assessed at
120 hpf. Similar to the control group, retinas from the $50 \mathrm{mg} / \mathrm{L}$ and $100 \mathrm{mg} / \mathrm{L}$ exposed groups did not show abnormal structural or cellular morphology (Figure 3A). For neural differentiation, three antibodies, namely, Zpr1, Zpr3 and Zn12, were used to label cones, rods and ganglion cells, respectively. Zpr1-positive and Zpr3positive cells were strongly detected in the outer nuclear layer (ONL), while $\mathrm{Zn} 12$-positive cells were found in the ganglion cell layer in all three groups (Figure 3B). To quantify this finding, we analyzed the fluorescence areas of the positive cells. There was no significant difference among groups (Figure 3C; ANOVA, $p>0.05$ ). To evaluate the hepatotoxicity of NPMOF, liver development was examined by HE staining and whole-mount in situ hybridization using the hepatocytespecific mRNA probe ceruloplasmin (cp) at $120 \mathrm{hpf}$. Images of HE staining showed that the liver was formed by well-delineated polygonal cells with abundant cytoplasm and a prominent nucleus in larvae from either the control or NPMOF-exposed groups (Figure 3D). The results of in situ hybridization showed that 
A Control

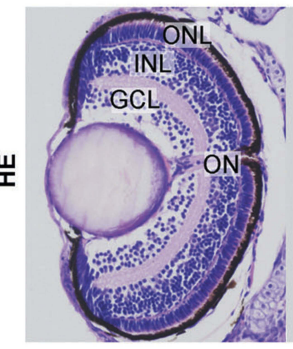

C
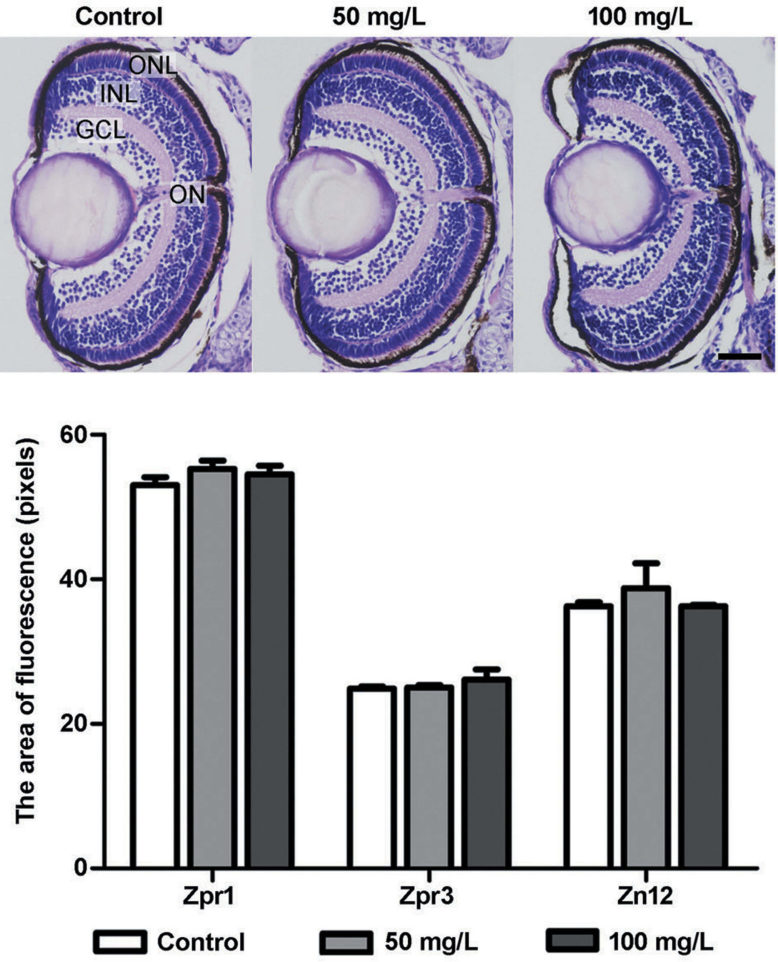

B

Control

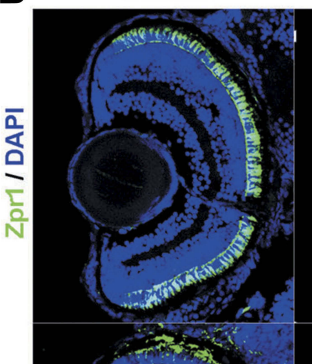

$50 \mathrm{mg} / \mathrm{L}$ $100 \mathrm{mg} / \mathrm{L}$
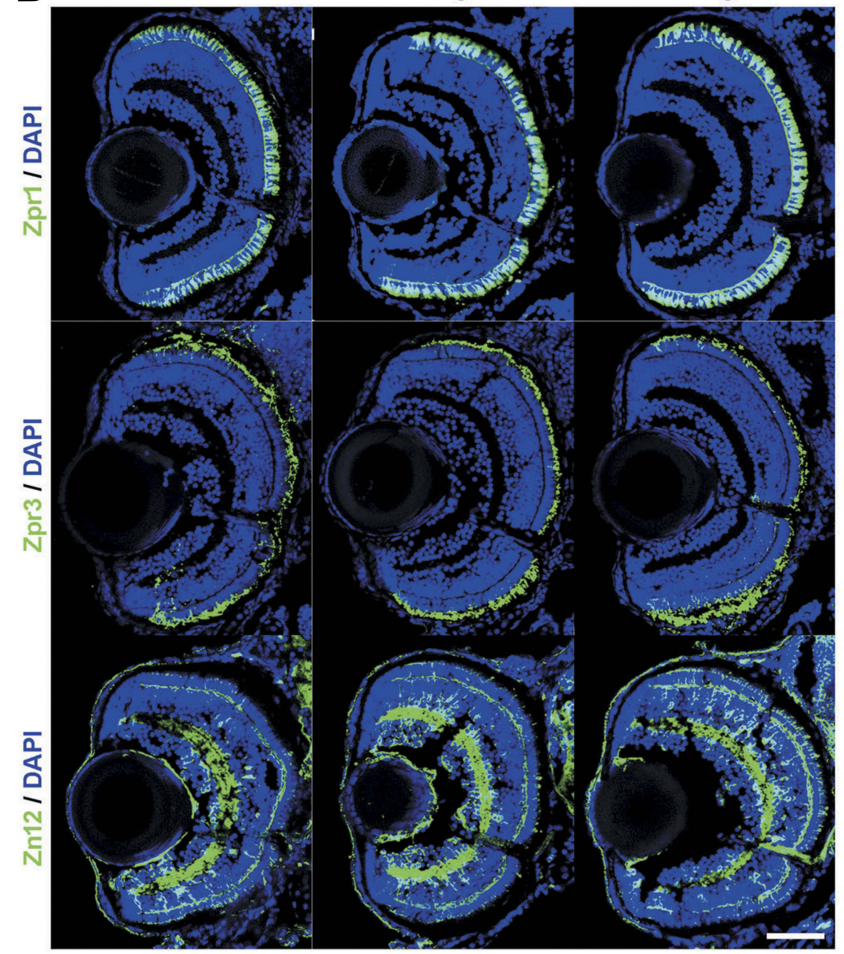

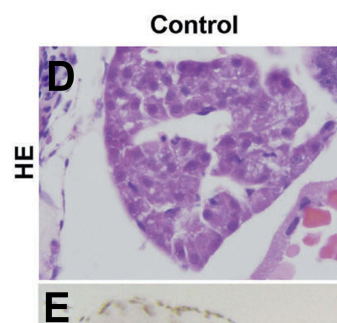

E

8

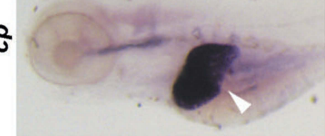

$50 \mathrm{mg} / \mathrm{L}$
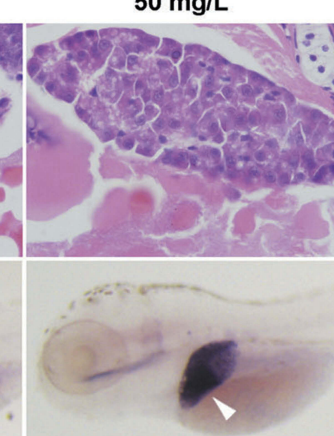

$100 \mathrm{mg} / \mathrm{L}$
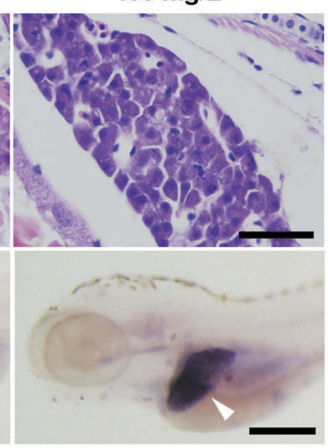
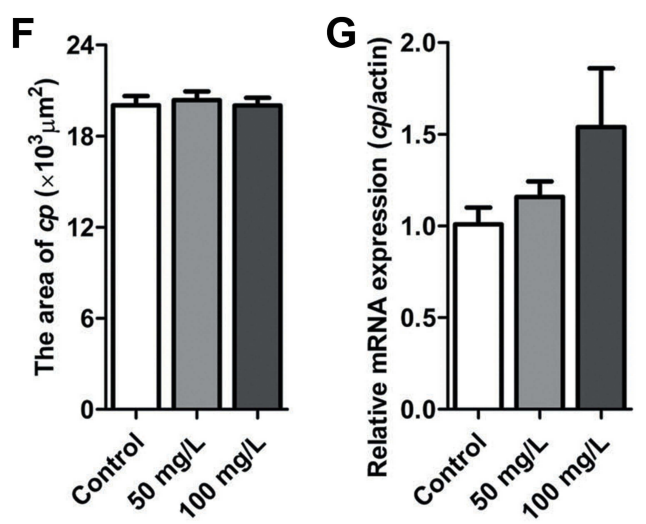

Figure 3 The histology of the retina and liver following NPMOF exposure. (A and B) Sections taken from the retina at I20 hpf. (A) HE staining; (B) ZprI, Zpr3 and Zn I2 staining. (C) Statistical analysis of Zprl-positive, Zpr3-positive and Zn I2-positive areas among the control, $50 \mathrm{mg} / \mathrm{L}$ and $100 \mathrm{mg} / \mathrm{L}$ exposed groups (ANOVA, p>0.05). (D) HE staining of the liver in the control, $50 \mathrm{mg} / \mathrm{L}$ and $100 \mathrm{mg} / \mathrm{L}$ groups. (E) Images of in situ hybridization. The expression of riboprobe ceruloplasmin (cp) $\mathrm{mRNA}$ is shown with arrowheads. (F) Statistical analysis of the liver size among the three groups (ANOVA, p>0.05). (G) The expression of cp. Dorsal is up and rostral is left in (E). Scale bar in (A) $20 \mu \mathrm{m}$; (B): $50 \mu \mathrm{m}$; (D): $20 \mu \mathrm{m}$; (E): $200 \mu \mathrm{m}$.

Abbreviations: ONL, outer nuclear layer; INL, inner nuclear layer; GCL, ganglion cell layer; ON, optic nerve.

$c p$-expressing cells were located posterior to the heart and lateral to the intestine (Figure 3E, arrowheads); meanwhile, the size of the livers was very similar in larvae from all three groups (Figure 3F; ANOVA, $p>0.05$ ). We also examined the expression level of $c p$ by qRT-PCR. There was no significant difference among the three groups (Figure 3G; ANOVA, $p>0.05$ ). These data indicate that exposure to NPMOF had little impact on neural differentiation and liver development.

\section{Evaluation of the Inflammatory Response Following NPMOF Exposure}

To verify whether NPMOF exposure could trigger an inflammatory response and reactive oxygen species (ROS), transgenic zebrafish $\mathrm{Tg}$ (lys $C$ :EGFP)were also exposed to the same NPMOF solution as the $\mathrm{AB}$ larvae. lys $C$ expression was reported to specifically label the neutrophils. ${ }^{30}$ In the process of the inflammatory response, neutrophils were recruited to the inflammatory site. ${ }^{31}$ At $96 \mathrm{hpf}$ and 120 
hpf, the number of neutrophils in the ocular (Figure 4A, circle) and hepatic regions (Figure 4C, square) was counted, and no significant difference was found among the three groups (Figure 4B and D; ANOVA, $p>0.05$ ). Tumor necrosis factor-alpha $(t n f \alpha)$ is a pleiotropic inflammatory cytokine, and superoxide dismutase $1(\operatorname{sod} 1)$ is an oxidoreductase that limits the detrimental effects of ROS. ${ }^{32,33}$ The expression of thf $\alpha$ and sod 1 was quantified by qRT-PCR at $120 \mathrm{hpf}$. The results showed that the expression was non-significant among the three groups (Figure 4E and F; ANOVA, $p>0.05)$. The negative results demonstrated that NPMOF did not trigger an inflammatory response. Hence, the larvae were in normal phenotypes with well-developed livers and fully differentiated retinas. As a drug carrier, the nano-scale size and low biotoxicity of NPMOF ensured its bioavailability and biocompatibility.

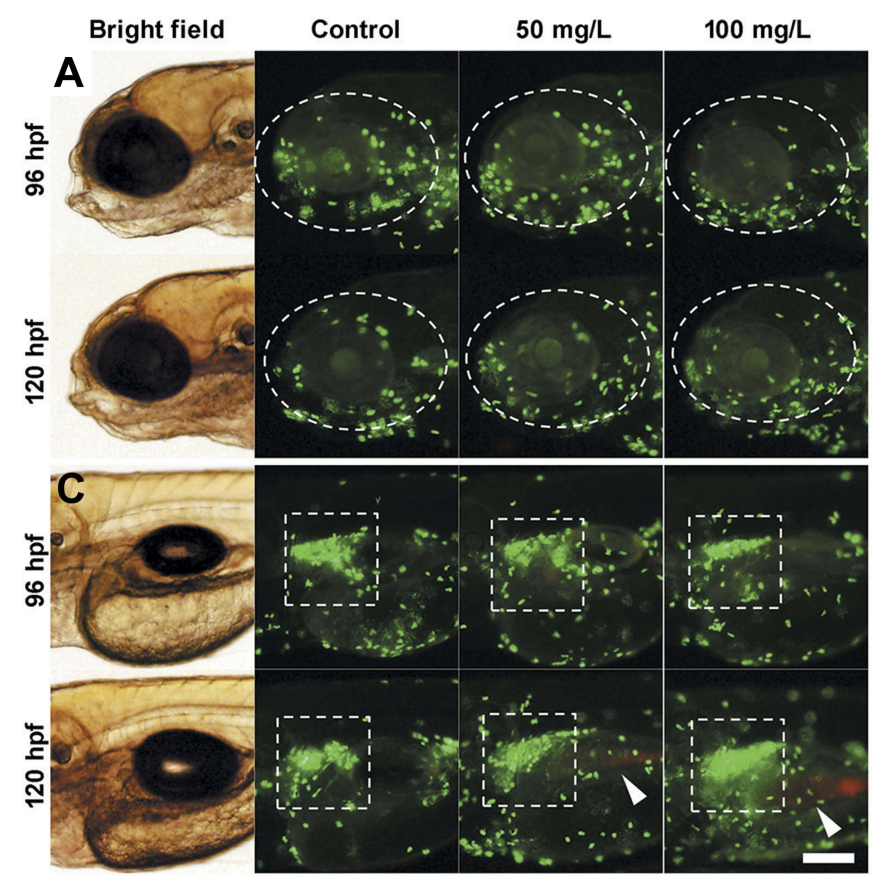

E

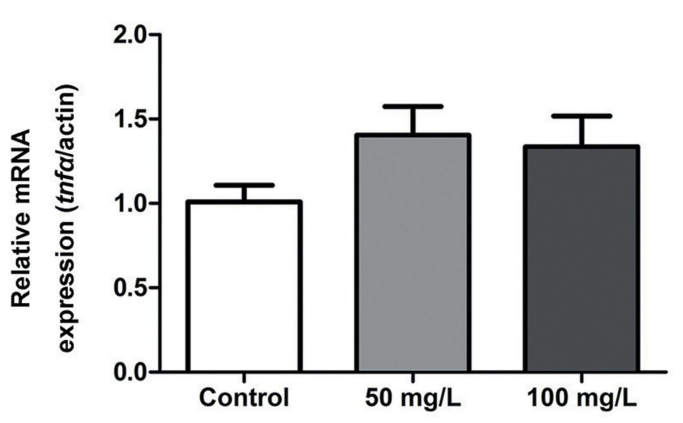

\section{NPMOF Retention in the Retina and} Uptake-Release Properties of MPS-NPMOF Intense light exposure to adult zebrafish specifically led the apoptosis of rods and cones and served as an excellent model to study photoreceptor regeneration. ${ }^{34}$ In this study, we used this model to investigate the distribution and retention time of NPMOF in retinas and therapeutic effect of the NPMOF loading systems. Following intravitreous injection of NPMOF, cryosections from $1 \mathrm{dpl}$ to $7 \mathrm{dpl}$ were performed. Time-lapse red fluorescence signals were found in the ON, choroid layer (CL) and retina, particularly in the ONL, photoreceptor layer (PL) and retinal pigment epithelium (RPE) layers (Figure 5A, arrowheads; Supplementary Figure 1a), suggesting that NPMOF diffused rapidly through the vitreous body, reached the photoreceptor and RPE layers along with the optic nerve and passed into the
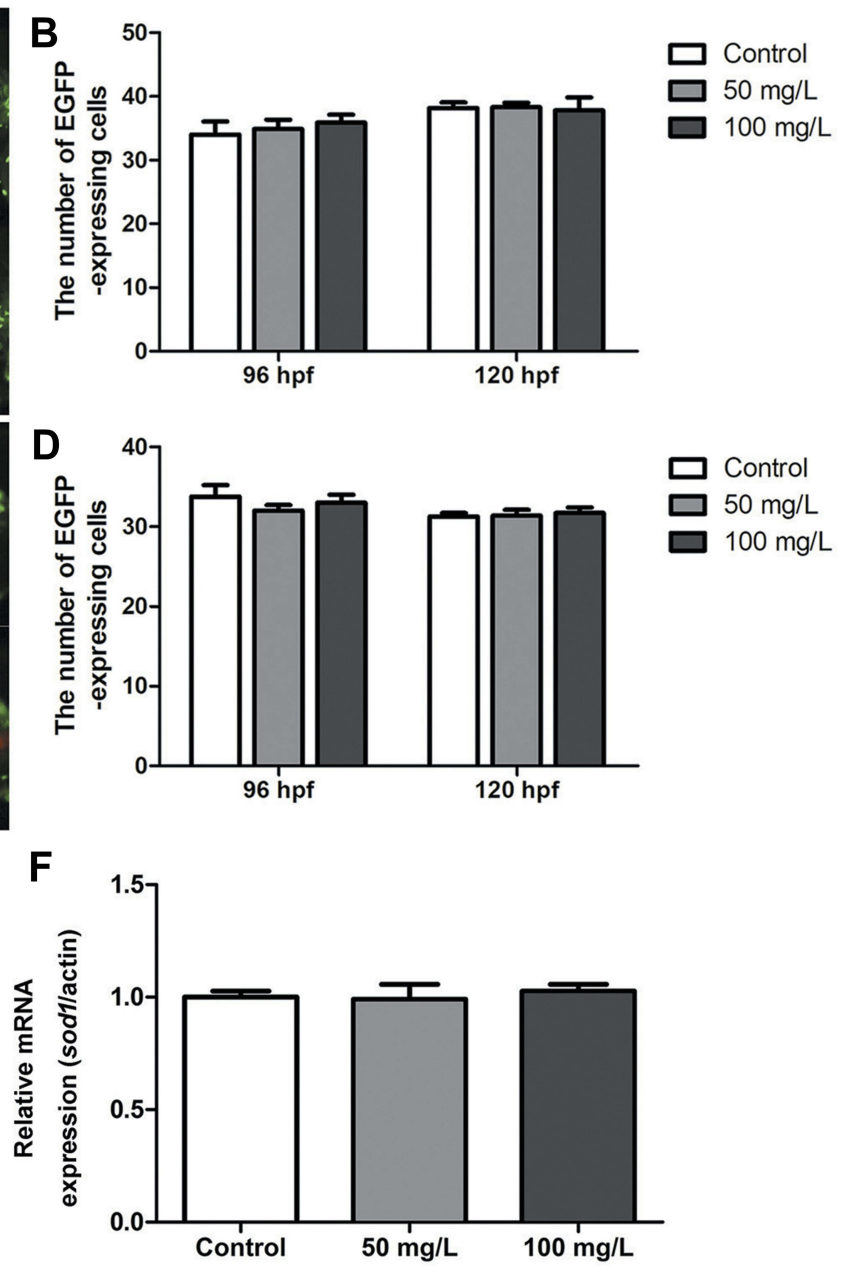

Figure 4 Inflammatory response following NPMOF exposure. (A and $\mathbf{C}$ ) The distribution of neutrophils in the (A) ocular region and (C) hepatic region. (B and $\mathbf{D})$ Statistical analysis of the number of neutrophils in the ocular (A, circle) and hepatic regions (C, square), respectively. The red fluorescence is emitted by NPMOF in the gastrointestinal tract $(\mathbf{C}$, arrowheads). (E and $\mathbf{F})$ The expression of tnf $\alpha$ and sodl. Dorsal is up and rostral is left in $(\mathbf{A})$ and $(\mathbf{C})$. Scale bar in $(\mathbf{A})$ : I00 $\mu \mathrm{m}$. 


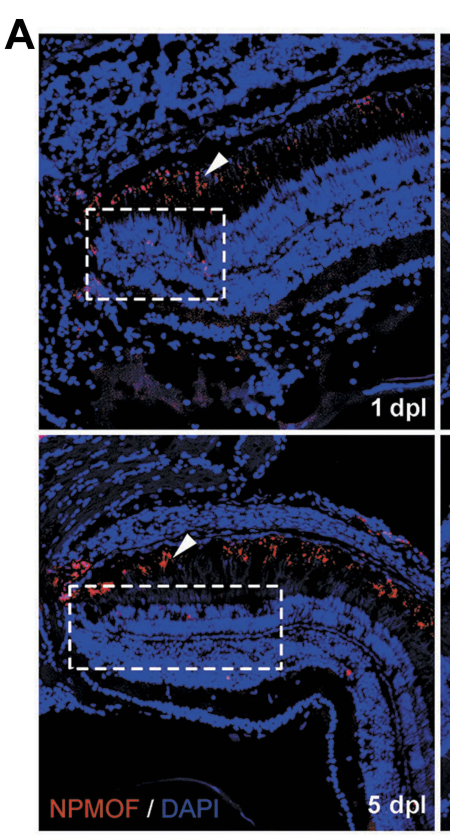

C

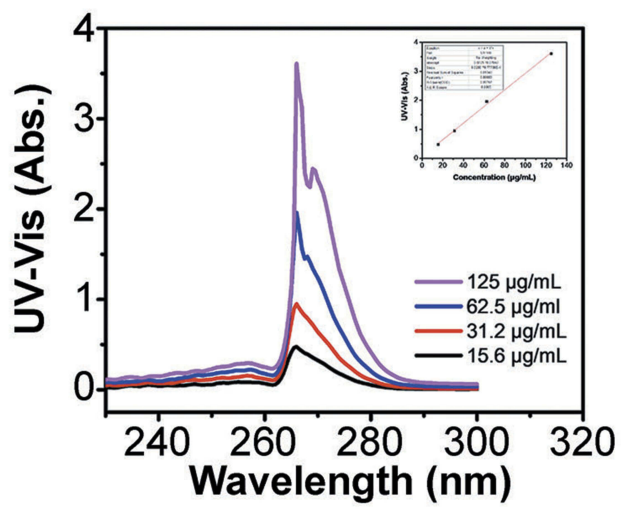

E

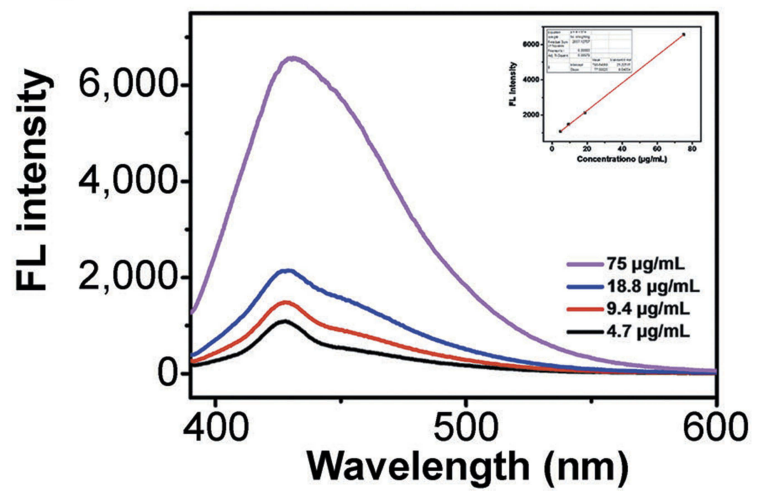

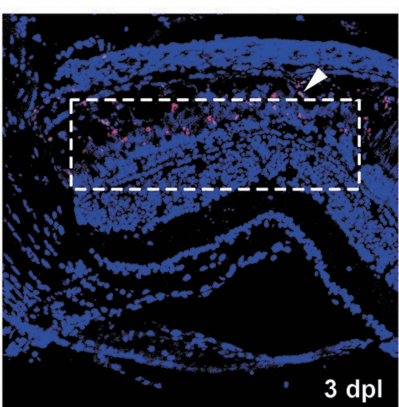


D

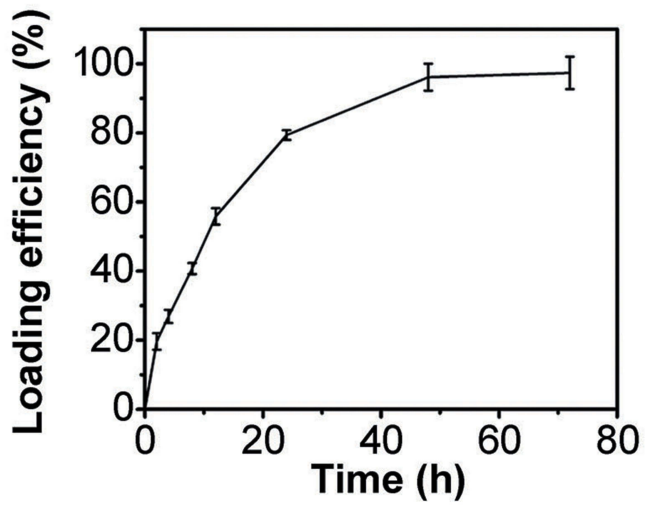

$\mathbf{F}$

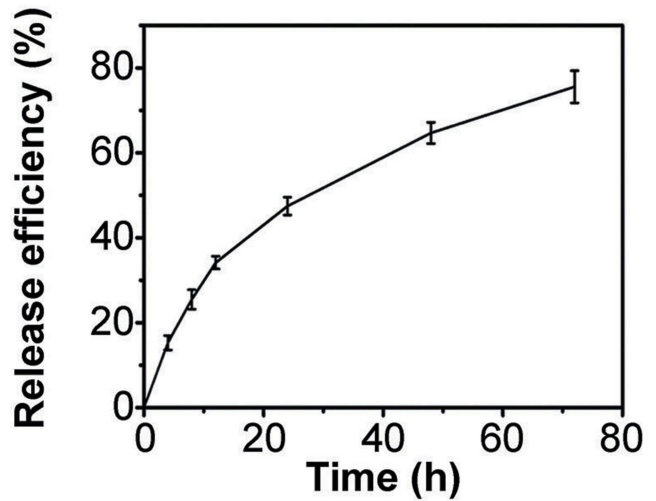

Figure 5 Distribution of NPMOF in the retina from I to 7 days post lesion and the loading efficiency and release efficiency for MPS in NPMOF. (A) The distribution of NPMOF (arrowheads) around the lesioned area (dotted rectangles) in the retina from I to $7 \mathrm{dpl}$. (B) The statistical analysis of NPMOF in the retina (ANOVA, * $<<0.05$ ). (C and D) The UV-Vis absorbance (insert, standard curve) of MPS in DMF and its loading performance. (E and F) The fluorescence (insert, standard curve) of MPS in $5 \%$ hyaluronic acid and its release performance. Scale bar in (A): $50 \mu \mathrm{m}$.

Abbreviations: $\mathrm{CL}$, choroid layer; RPE, retinal pigment epithelium; ONL, outer nuclear layer; INL, inner nuclear layer; GCL, ganglion cell layer; ON, optic nerve.

choroid layer to be cleaned (Figure 5A). To identify the amount of NPMOF around the lesioned site (Figure 5A, dotted rectangles), the area of red fluorescence was measured at each time point. It increased sharply and reached its peak at $2 \mathrm{dpl}$, before decreasing gradually until $7 \mathrm{dpl}$ (Figure 5B). In particular, the amount of NPMOF at 2 
dpl was significantly higher than that at 1, 4, 5, 6 and $7 \mathrm{dpl}$, and the amount of NPMOF at $3 \mathrm{dpl}$ was also above that at 1 , 5,6 and $7 \mathrm{dpl}$ (Figure 5B; ANOVA, ${ }^{*} p<0.05$ ). Statistics of the amount of NPMOF in different layers was shown in Supplementary Figure 1b. Within two days, more fluorescent signals were found in RPE and PL (Supplementary Figure $1 \mathrm{~b}$, grey columns; ANOVA, ${ }^{*} p<0.05$ ). At 3 days post lesion, the amount reached the peak in CL (Supplementary Figure 1b, white columns; ANOVA, ${ }^{*} p<0.05$ ). These data suggest that NPMOF can be retained in the retina for 7 days and that the concentration is enriched at 2 and $3 \mathrm{dpl}$.

Figure $5 \mathrm{C}$ show the loading property of MPS. The loading efficiency was $97.3 \%(\mathrm{w} / \mathrm{w})$ at $72 \mathrm{~h}$ (Figure 5D). The high loading efficiency of MPS was attributed to the interconnected 3D pore geometry and large channel size of NPMOF. 5\% hyaluronic acid was used to imitate vitreous body. The releasing property of MPS in 5\% hyaluronic acid is given in Figure 5E. MPS in NPMOF was released at a moderate rate in 5\% hyaluronic acid $(75.6 \%$ at $72 \mathrm{~h}$; Figure 5F). Currently, intravitreous injection is the most common procedure in the treatment of vitreoretinal diseases. ${ }^{7}$ Compared to systemic administration, intravitreous injection can minimize the potential systemic adverse effects. However, the repetitive injections may lead to unfavorable outcomes, including subconjunctival hemorrhage, intraocular pressure rise, floaters and infection. ${ }^{7,35}$ Therefore, the long-standing drug delivery system is an ideal approach. After intravitreous injection, NPMOF distributed throughout the retina quickly and remained in the retina for 7 days. In vitro experiments also showed that MPS-NPMOF released moderately in 5\% hyaluronic acid. Both of the above results ensured the sustained effect.

\section{Photoreceptor Regeneration and Recovery of Visual Function}

To explore whether the injection of MPS-NPMOF had any impact at the lesioned site, the morphology of retinas from the PBS, MPS and MPS-NPMOF groups was evaluated at 1 $\mathrm{dpl}, 3 \mathrm{dpl}$ and $7 \mathrm{dpl}$. At $1 \mathrm{dpl}$, nuclear debris appeared in the outer segment layer (OSL), which suggested that photoreceptors were exfoliated from the ONL to the OSL (Supplementary Figure 2a, dotted rectangles). At $3 \mathrm{dpl}$, the nuclear layer structure of the retina was disrupted, and cells in the cone outer nuclear layer (cONL) were completely interrupted (Supplementary Figure 2a, arrowheads). Interestingly, the MPS-NPMOF group had a tighter arrangement and shorter defect range of retinal cells compared to the other groups (Supplementary Figure $2 \mathrm{~b}$ and $\mathrm{c}$; ANOVA, $\left.{ }^{*} \mathrm{p}<0.05\right)$. Moreover, long spindle-shaped cells appeared in the nuclear layer at $3 \mathrm{dpl}$ (Supplementary Figure 2a, arrows). At $7 \mathrm{dpl}$, the lesioned site was filled with cells in all three groups (Supplementary Figure 2a).

Based on the results of HE staining, retinas from PBS, MPS and MPS-NPMOF groups were immunolabeled by Zpr1 and Zpr3 antibodies at $3 \mathrm{dpl}$ and $7 \mathrm{dpl}$ to further investigate the degeneration and regeneration of cones and rods, respectively. In the PBS and MPS groups at $3 \mathrm{dpl}$, the Zpr1-positive cells were absent or disorganized in the lesioned retina, and the ONL of the lesion site was thinner than that in the unlesioned region (Figure 6A). In the MPSNPMOF group, the arrangement of Zpr1-positive cells was also completely interrupted at $3 \mathrm{dpl}$, whereas the shorter defect range meant that more cones were present than in the PBS and MPS groups per 300 microns (Figure 6A and C; ANOVA, $\left.{ }^{*} p<0.05\right)$. Zpr3-positive cells had a similar performance, showing massive exfoliation in the PBS group, partial exfoliation in the MPS group and slight disorganization in the MPS-NPMOF group (Figure 6A). The MPSNPMOF group had more residual rods in the lesioned site (Figure 6D; ANOVA, ${ }^{*} p<0.05$ ). These data indicate that only minor ablation of cones and rods were observed in the MPSNPMOF group at $3 \mathrm{dpl}$. In the PBS and MPS groups at $7 \mathrm{dpl}$, Zpr1-positive cells filled in the OSL of the lesioned site with a loose pattern, while Zpr1 was strongly and continuously expressed in the MPS-NPMOF group (Figure 6B). Zpr3 was strongly expressed in all three groups at $7 \mathrm{dpl}$ (Figure 6B), indicating that rods were well regenerated. At this time point, no significant difference was found in the numbers of Zpr1positive or Zpr3-positive cells among the three groups (Figure 6E and F; ANOVA, $p>0.05$ ). To determine the corresponding visual function, optomotor response (OMR) analysis was performed. Both the positive proportion of distance and positive proportion of time in the MPS-NPMOF group were close to those in the normal group, while the PBS and MPS groups showed significant decreases (Figure 6G and H; ANOVA, $\left.{ }^{*} p<0.05\right)$. These data suggest that retinas in the MPS-NPMOF group gained better differentiated cones and visual function at $7 \mathrm{dpl}$. MPS had the ability to protect the retina from damage. ${ }^{5}$ We administrated MPS-NPMOF via intravitreous injection. It avoided the lower efficiency and adverse effects caused by systemic administration and prolonged the retention time of the drug. ${ }^{36,37}$ That's why retinas revealed smaller scale of lesioned site, milder injury and faster recovery following MPS-NPMOF injection. The 

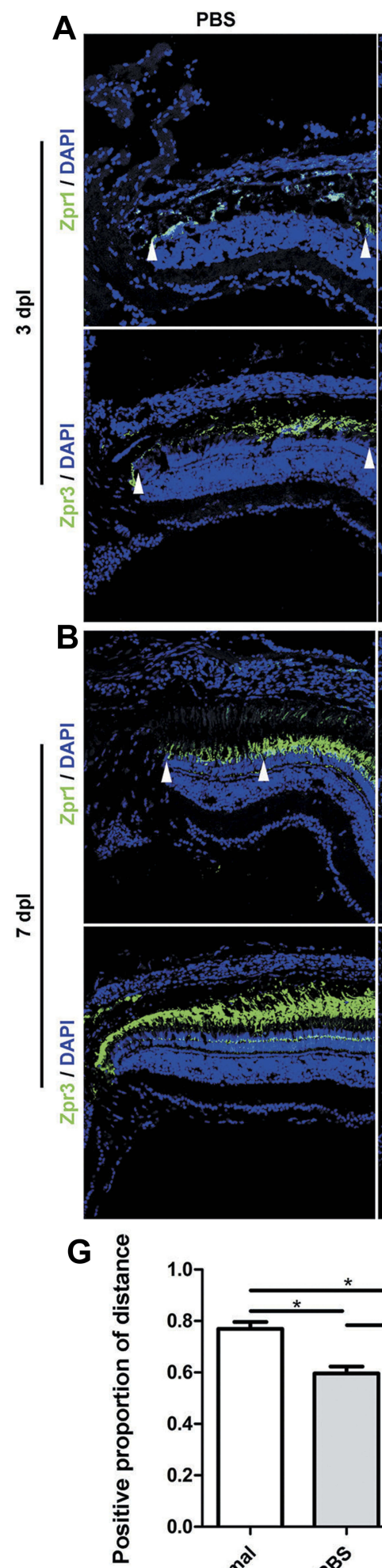
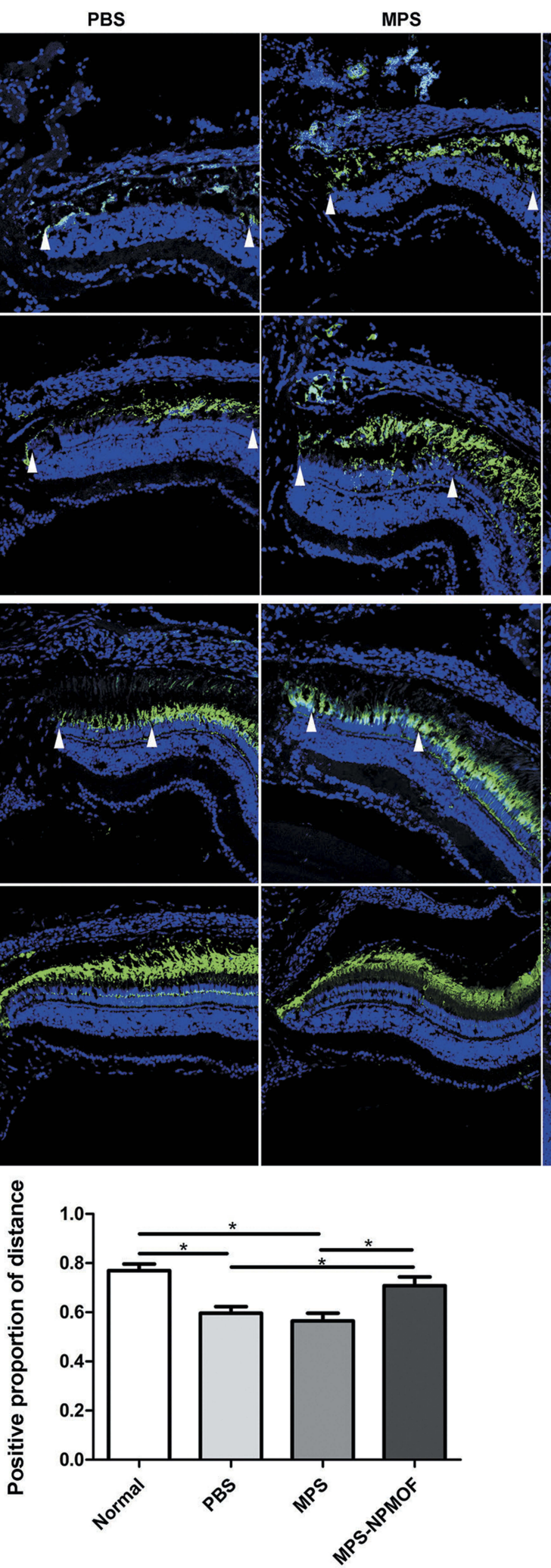

MPS-NPMOF
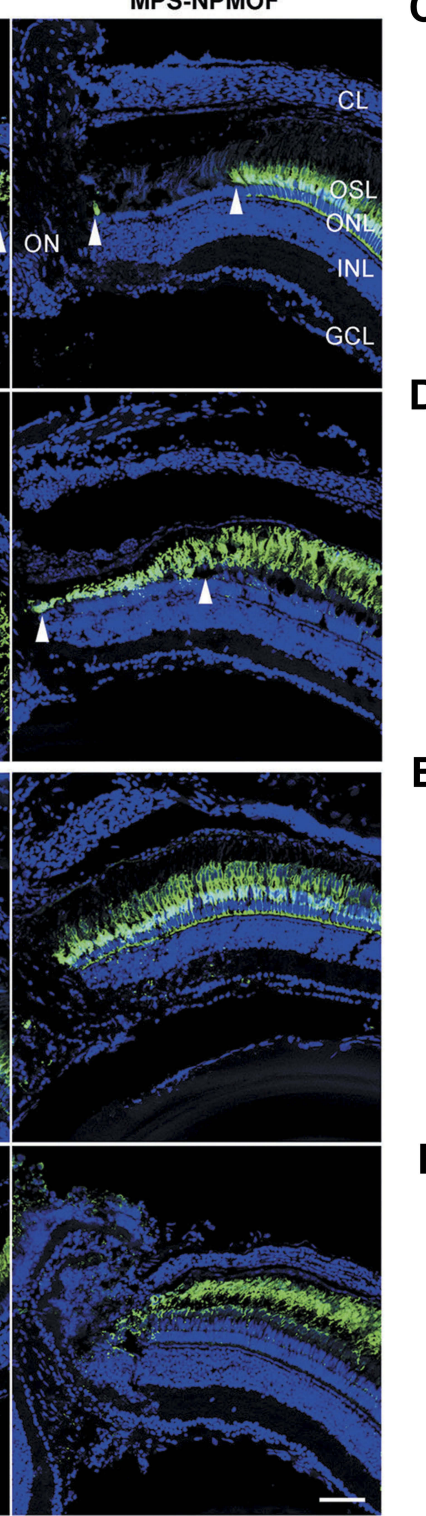

C

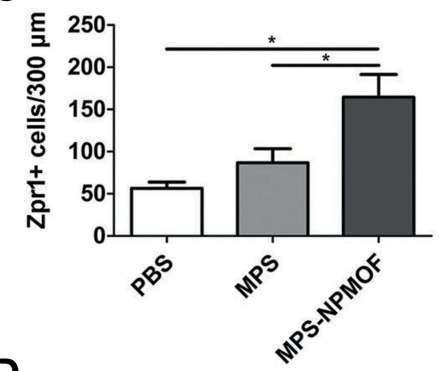

D



E
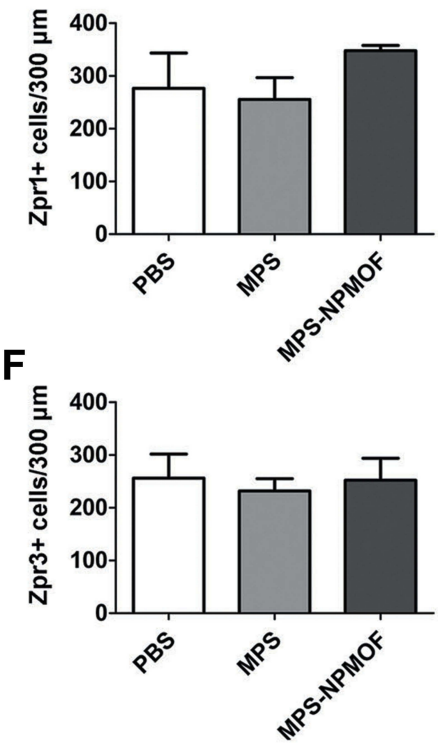

Figure 6 Degeneration-regeneration of photoreceptors and changes in visual function following treatment with MPS-NPMOF. (A and B) Zprl and Zpr3 staining in sections taken from retinas in the PBS, MPS and MPS-NPMOF groups at 3 and $7 \mathrm{dpl}$. Note that retinas in the PBS and MPS groups have a loose arrangement of ZprI-positive cells at lesioned sites (arrowheads) at $7 \mathrm{dpl}$. (C and D) Quantification of the number of cones and rods per 300 microns in the lesioned retina at 3 dpl. More photoreceptors remained in the MPS-NPMOF group compared to the PBS and MPS groups (ANOVA, ${ }^{*} p<0.05$ ). (E and F) Quantification of the number of cones and rods per 300 microns at $7 \mathrm{dpl}$. There are no significant differences among the three groups (ANOVA, $p>0.05$ ). ( $\mathbf{G}$ and $\mathbf{H}$ ) The statistical analyses of the positive proportion of distance and positive proportion of time at $7 \mathrm{dpl}$. Note that the significant decrease in the PBS and MPS groups (ANOVA, * $p<0.05)$. Scale bar in $(\mathbf{A})$ and $(\mathbf{B}): 50 \mu \mathrm{m}$.

Abbreviations: $\mathrm{CL}$, choroid layer; OSL, outer segment layer; ONL, outer nuclear layer; INL, inner nuclear layer; GCL, ganglion cell layer; ON, optic nerve. 
NPMOF-related drug delivery system was demonstrated to be effective.

\section{Cell Proliferation at the Lesion Site}

During the regeneration process of retina, the proliferating cells were derived from Müller glia and recognized by PCNA. ${ }^{38}$ To investigate the underlying mechanisms of NPMOF-MPS promoting recovery, the anti-PCNA antibody was used to identify precursor cells and evaluate cell proliferation, while the expression of asclla and sox 2 was examined. At $1 \mathrm{dpl}$, scattered PCNA-positive cells gathered in clusters in the INL in retinas from the PBS, MPS and MPSNPMOF groups (Figure 7A). At 2 dpl, the increasing PCNApositive cells were found to have a long spindle shape, extending from the INL to the ONL (Figure 7A). At $3 \mathrm{dpl}$, the compact proliferating cells in the PBS and MPS groups
A

A

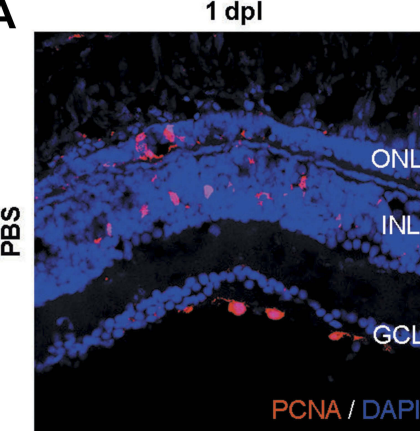

ֻ
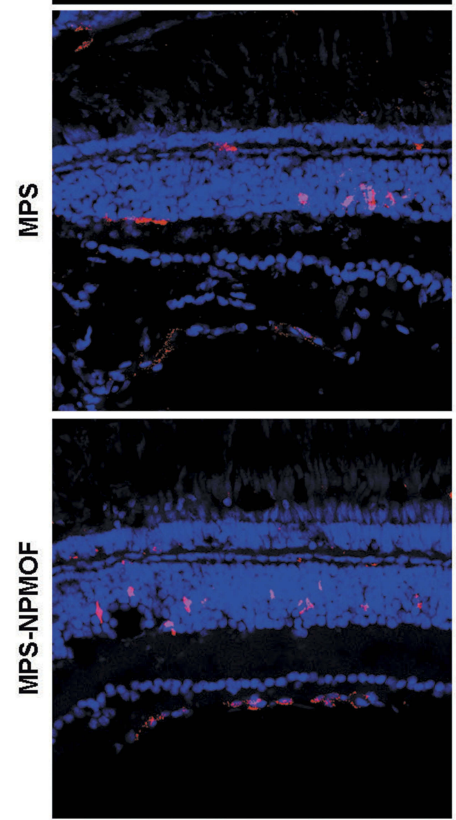

B

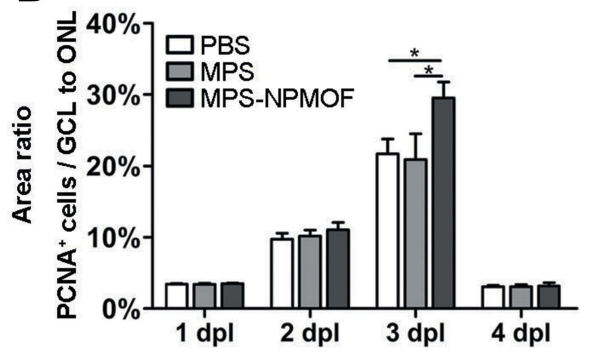

$2 \mathrm{dpl}$
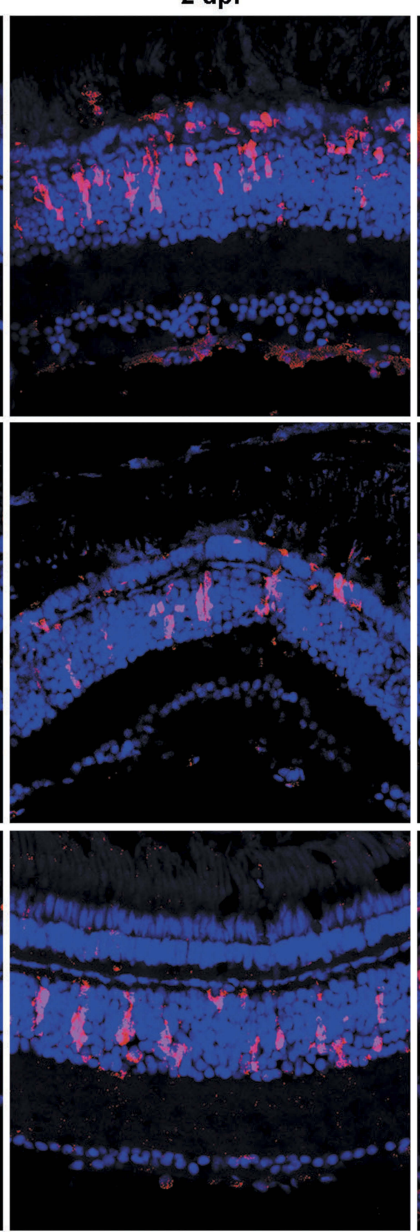

C

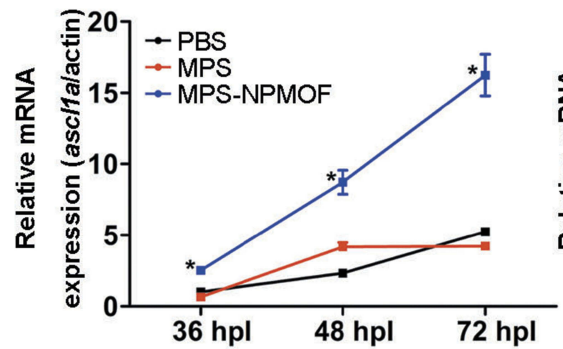

$3 \mathrm{dpl}$
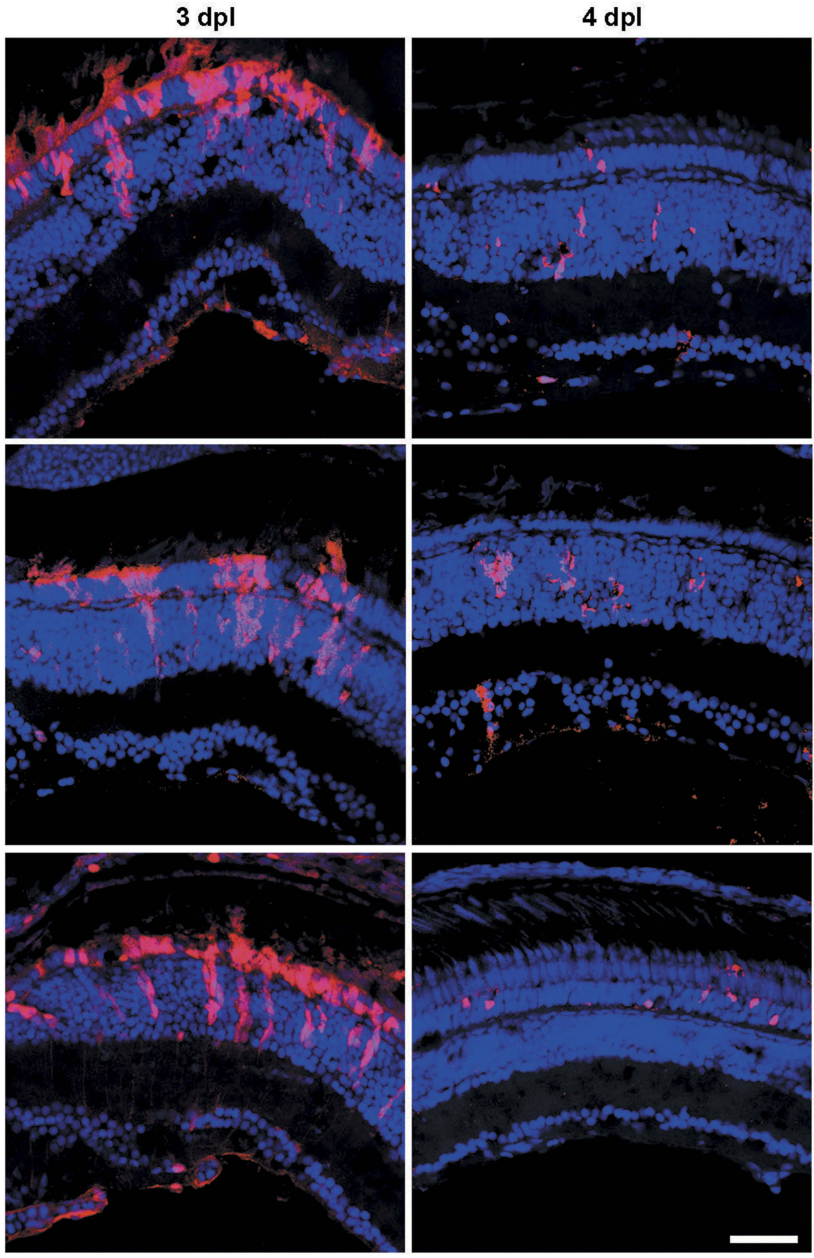

D



Figure 7 Cell proliferation of light-lesioned retina following injection of MPS-NPMOF. (A) PCNA staining in sections taken from retinas at I, 2, 3 and 4 dpl. (B) Statistical analysis of the percentage of PCNA-positive cells from GCL to ONL. There was a significantly higher percentage in the MPS-NPMOF group than in the PBS and MPS groups at $3 \mathrm{dpl}$ (ANOVA, *p<0.05). (C and D) The expression of ascll $a$ and sox2 mRNA. Note that ascll $a$ was increased in the MPS-NPMOF group at 36, 48 and $72 \mathrm{hpl}$, while sox2 was increased at 48 and $72 \mathrm{hpl}($ ANOVA, $* p<0.05)$. Scale bar in $(\mathbf{A}): 20 \mu \mathrm{m}$.

Abbreviations: ONL, outer nuclear layer; INL, inner nuclear layer; GCL, ganglion cell layer. 
had almost finished their migration to the ONL. However, the proliferating cells in the MPS-NPMOF group not only clustered in the ONL but also distributed in the INL (Figure 7A). At $4 \mathrm{dpl}$, the number of proliferating cells decreased in all three groups (Figure 7A). We then scored the percentage of PCNA-positive cells (the area of PCNA-positive cells/the total area from GCL to ONL) to quantify these findings. At $3 \mathrm{dpl}$, there was a significantly higher percentage of PCNApositive cells in the retinas of the MPS-NPMOF group than those from the PBS and MPS groups (Figure 7B; ANOVA, $\left.{ }^{*} p<0.05\right)$. Since asclla and sox 2 genes were required for the Müller glia's reprogramming and proliferation, ${ }^{15,39}$ we performed qRT-PCR to examine their expression. Compared with the PBS and MPS groups, the MPS-NPMOF group showed an increased expression of asclla from $36 \mathrm{hrs}$ post lesion (hpl) (Figure 7C; ANOVA, ${ }^{*} p<0.05$ ), while a statistically higher expression of sox 2 appeared from $48 \mathrm{hpl}$ (Figure 7D; ANOVA, ${ }^{*} p<0.05$ ). The above results indicate that MPS-NPMOF promotes Müller glia proliferation and retinal regeneration.

\section{Conclusion}

In summary, the novel synthesized nanoparticle, NPMOF, has excellent in vivo biocompatibility and low biotoxicity, making it an ideal skeleton to prepare drug delivery systems. By intraocular injection, MPS-NPMOF releases at the lesion site continuously and exerts the therapeutic effects of MPS, including defending the stress-induced apoptosis of retina after light injury and promoting photoreceptor regeneration. Intraocular injection of the loaded-NPMOF delivery system provides a promising approach to treat ocular posterior segment diseases, such as solar retinopathy, diabetic retinopathy and age-related macular degeneration.

\section{Acknowledgments}

This work was supported by Chinese National Natural Science Foundation (No. 81671179 and 81971739), the National Key R\&D Program of China (No. 2017YFA0103201), Tianjin Talent Innovation Group of 131, Bureau of Personnel, CAMS Innovation Fund for Medical Sciences (No. 2016I2M-1-017 \& 2017-I2M-B\&R-13), and the Fundamental Research Funds for the Central Universities (Nankai University No. 63191154). The authors thank Dr. Shaohua Yao (State Key Laboratory of Biotherapy and Cancer Center, West China Hospital, Sichuan University) for providing transgenic zebrafish Tg (lysC:EGFP).

\section{Disclosure}

The authors report no conflicts of interest in this work.

\section{References}

1. Contin MA, Benedetto MM, Quinteros-Quintana ML, Guido ME. Light pollution: the possible consequences of excessive illumination on retina. Eye. 2016;30(2):255-263. doi:10.1038/eye.2015.221

2. Blanco-Sanchez B, Clement A, Phillips JB, Westerfield M. Zebrafish models of human eye and inner ear diseases. Methods Cell Biol. 2017;138:415-467. doi:10.1016/bs.mcb.2016.10.006

3. Behar-Cohen F, Zhao M. Corticosteroids and the retina: a role for the mineralocorticoid receptor. Curr Opin Neurol. 2016;29(1):49-54. doi:10.1097/WCO.0000000000000284

4. Zhao M, Bousquet E, Valamanesh F, et al. Differential regulations of AQP4 and Kir4.1 by triamcinolone acetonide and dexamethasone in the healthy and inflamed retina. Invest Ophthalmol Vis Sci. 2011;52 (9):6340-6347. doi:10.1167/iovs.11-7675

5. Sulaiman RS, Kadmiel M, Cidlowski JA. Glucocorticoid receptor signaling in the eye. Steroids. 2018;133:60-66. doi:10.1016/j. steroids.2017.11.002

6. Fini ME, Schwartz SG, Gao X, et al. Steroid-induced ocular hypertension/glaucoma: focus on pharmacogenomics and implications for precision medicine. Prog Retin Eye Res. 2017;56:58-83. doi:10.1016/ j.preteyeres.2016.09.003

7. Gupta A, Sun JK, Silva PS. Complications of intravitreous injections in patients with diabetes. Semin Ophthalmol. 2018;33(1):42-50. doi:10.1080/08820538.2017.1353811

8. Huang D, Chen YS, Green CR, Rupenthal ID. Hyaluronic acid coated albumin nanoparticles for targeted peptide delivery in the treatment of retinal ischaemia. Biomaterials. 2018;168:10-23. doi:10.1016/j. biomaterials.2018.03.034

9. Sahoo SK, Dilnawaz F, Krishnakumar S. Nanotechnology in ocular drug delivery. Drug Discov Today. 2008;13(3-4):144-151. doi:10.1016/j.drudis.2007.10.021

10. Zheng H, Zhang Y, Liu L, et al. One-pot synthesis of metal-organic frameworks with encapsulated target molecules and their applications for controlled drug delivery. J Am Chem Soc. 2016;138(3):962-968. doi:10.1021/jacs.5b11720

11. Liu W, Wang YM, Li YH, et al. Fluorescent imaging-guided chemotherapy-and-photodynamic dual therapy with nanoscale porphyrin metal-organic framework. Small. 2017;13(17):1603459. doi:10.1002/Smll.201603459

12. Westerfield M. The Zebrafish Book: A Guide for the Laboratory Use of zebrafish (Brachydanio Rerio). Eugene (OR): University of Oregon Press: M. Westerfield; 2007.

13. Wang YJ, Cai SJ, Cui JL, Chen Y, Tang X, Li YH. Correlation between photoreceptor injury-regeneration and behavior in a zebrafish model. Neural Regen Res. 2017;12(5):795-803. doi:10.4103/1673-5374.206651

14. Rosch S, Johnen S, Muller F, Pfarrer C, Walter P. Correlations between ERG, OCT, and anatomical findings in the rd10 mouse. J Ophthalmol. 2014;2014:874751. doi:10.1155/2014/874751

15. Gorsuch RA, Lahne M, Yarka CE, Petravick ME, Li J, Hyde DR. Sox 2 regulates Muller glia reprogramming and proliferation in the regenerating zebrafish retina via Lin28 and Asclla. Exp Eye Res. 2017;161:174-192. doi:10.1016/j.exer.2017.05.012

16. Nelson CM, Ackerman KM, O'Hayer P, Bailey TJ, Gorsuch RA, Hyde DR. Tumor necrosis factor-alpha is produced by dying retinal neurons and is required for Muller glia proliferation during zebrafish retinal regeneration. J Neurosci. 2013;33(15):6524-6539. doi:10.1523/ JNEUROSCI.3838-12.2013

17. Thisse C, Thisse B. High-resolution in situ hybridization to whole-mount zebrafish embryos. Nat Protoc. 2008;3(1):59-69. doi:10.1038/nprot.2007.514 
18. Li J, Liu QT, Chen Y, et al. Involvement of 5-HT1A receptors in the anxiolytic-like effects of quercitrin and evidence of the involvement of the monoaminergic system. Evid Based Complement Alternat Med. 2016;2016:6530364. doi:10.1155/2016/5040528

19. Kopp R, Legler J, Legradi J. Alterations in locomotor activity of feeding zebrafish larvae as a consequence of exposure to different environmental factors. Environ Sci Pollut Res Int. 2018;25 (5):4085-4093. doi:10.1007/s11356-016-6704-3

20. Maaswinkel H, Li L. Spatio-temporal frequency characteristics of the optomotor response in zebrafish. Vision Res. 2003;43(1):21-30. doi:10.1016/S0042-6989(02)00395-4

21. Gould CJ, Wiegand JL, Connaughton VP. Acute developmental exposure to 4-hydroxyandrostenedione has a long-term effect on visually-guided behaviors. Neurotoxicol Teratol. 2017;64:45-49. doi:10.1016/j.ntt.2017.10.003

22. Dutta A, Mondal J, Patra AK, Bhaumik A. Synthesis and temperature-induced morphological control in a hybrid porous iron-phosphonate nanomaterial and its excellent catalytic activity in the synthesis of benzimidazoles. Chemistry. 2012;18(42):13372-13378. doi:10.1002/chem.v18.42

23. Liu D, Huxford RC, Lin W. Phosphorescent nanoscale coordination polymers as contrast agents for optical imaging. Angew Chem Int Ed Engl. 2011;50(16):3696-3700. doi:10.1002/anie.v50.16

24. Minchin JE, Williams VC, Hinits Y, et al. Oesophageal and sternohyal muscle fibres are novel Pax3-dependent migratory somite derivatives essential for ingestion. Development. 2013;140 (14):2972-2984. doi:10.1242/dev.090050

25. Conte C, Costabile G, d'Angelo I, et al. Skin transport of PEGylated poly (epsilon-caprolactone) nanoparticles assisted by (2-hydroxypropyl)-betacyclodextrin. J Colloid Interface Sci. 2015;454:112-120. doi:10.1016/j. jcis.2015.05.010

26. Gell DA. Structure and function of haemoglobins. Blood Cells Mol Dis. 2018;70:13-42. doi:10.1016/j.bcmd.2017.10.006

27. Liang R, Ghaffari S. Advances in understanding the mechanisms of erythropoiesis in homeostasis and disease. Br J Haematol. 2016;174 (5):661-673. doi:10.1111/bjh.2016.174.issue-5

28. Hutchinson SA, Tooke-Locke E, Wang J, Tsai S, Katz T, Trede NS. Tb13 regulates cell cycle length during zebrafish development. Dev Biol. 2012;368(2):261-272. doi:10.1016/j.ydbio.2012.05.024
29. Fernandez-Murray JP, Prykhozhij SV, Dufay JN, et al. Glycine and Folate Ameliorate Models of Congenital Sideroblastic Anemia. PLoS Genet. 2016;12(1):e1005783. doi:10.1371/journal.pgen.1005783

30. Barros-Becker F, Lam PY, Fisher R, Huttenlocher A. Live imaging reveals distinct modes of neutrophil and macrophage migration within interstitial tissues. $J$ Cell Sci. 2017;130(22):3801-3808. doi:10.1242/jcs. 206128

31. Robertson AL, Holmes GR, Bojarczuk AN, et al. A zebrafish compound screen reveals modulation of neutrophil reverse migration as an anti-inflammatory mechanism. Sci Transl Med. 2014;6 (225):225ra229. doi:10.1126/scitranslmed.3007672

32. Felix LM, Vidal AM, Serafim C, et al. Ketamine induction of p53-dependent apoptosis and oxidative stress in zebrafish (Danio rerio) embryos. Chemosphere. 2018;201:730-739. doi:10.1016/j. chemosphere.2018.03.049

33. Lei XD, Sun Y, Cai SJ, Fang YW, Cui JL, Li YH. Role of tumor necrosis factor-alpha in zebrafish retinal neurogenesis and myelination. Int $J$ Ophthalmol. 2016;9(6):831-837. doi:10.18240/ ijo.2016.06.07

34. Rajaram K, Summerbell ER, Patton JG. Technical brief: constant intense light exposure to lesion and initiate regeneration in normally pigmented zebrafish. Mol Vis. 2014;20:1075-1084.

35. Writing Committee for the Multicenter Uveitis Steroid Treatment T, Follow-up Study Research G, Kempen JH, et al. Association between long-lasting intravitreous fluocinolone acetonide implant vs systemic anti-inflammatory therapy and visual acuity at 7 years among patients with intermediate, posterior, or panuveitis. JAMA. 2017;317 (19):1993-2005. doi:10.1001/jama.2017.5103

36. Jacob S, Nair AB. An updated overview with simple and practical approach for developing in vitro-in vivo correlation. Drug Dev Res. 2018;79(3):97-110. doi:10.1002/ddr.21427

37. Liu S, Liu X, Chen S, Xiao Y, Zhuang W. Oral versus intravenous methylprednisolone for the treatment of multiple sclerosis relapses: a meta-analysis of randomized controlled trials. PLoS One. 2017;12 (11):e0188644. doi:10.1371/journal.pone.0188644

38. Wan J, Goldman D. Retina regeneration in zebrafish. Curr Opin Genet Dev. 2016;40:41-47. doi:10.1016/j.gde.2016.05.009

39. Goldman D. Muller glial cell reprogramming and retina regeneration. Nat Rev Neurosci. 2014;15(7):431-442. doi:10.1038/nrn3723
International Journal of Nanomedicine

\section{Publish your work in this journal}

The International Journal of Nanomedicine is an international, peerreviewed journal focusing on the application of nanotechnology in diagnostics, therapeutics, and drug delivery systems throughout the biomedical field. This journal is indexed on PubMed Central, MedLine, CAS, SciSearch ${ }^{\mathbb{R}}$, Current Contents ${ }^{\mathbb{R}} /$ Clinical Medicine, $^{2}$
Journal Citation Reports/Science Edition, EMBase, Scopus and the Elsevier Bibliographic databases. The manuscript management system is completely online and includes a very quick and fair peer-review system, which is all easy to use. Visit http://www.dovepress.com/ testimonials.php to read real quotes from published authors 